\title{
One World, One Sky: Outreach in a Multicultural, Multilingual Metropolis
}

\author{
M. Reid \\ Dunlap Institute for Astronomy and Astrophysics, University of Toronto \\ email: mike.reid@utoronto.ca
}

As cities around the world grow more and more diverse, we must take this diversity into account in developing outreach activities and materials. The International Year of Astronomy in 2009 brought a lot of attention to the needs of underserved communities and developing countries, emphasizing the ideal of widespread access to astronomy outreach. Increasingly, however, we find that some of the same challenges facing underserved communities and developing countries are also present in modern metropolises. Conveniently, the linguistic and cultural diversity of our cities is more and more accurately reflected among the astronomy community. The diversity of the astronomical community itself creates opportunities for effective multicultural, multilingual outreach. The Dunlap Institute is located in Toronto, the largest city in Canada and one of the most diverse in the world. The Canadian emphasis on multiculturalism has produced in Toronto a unique blending of the world's cultures. According to Statistics Canada, nearly half of Torontonians are foreign-born and furthermore, half of Torontonians speak a language other than English as their mother tongue . Many of those whose first language is not English do not speak English at all, or not well enough to comfortably discuss astronomy in English. We have found that traditional forms of outreach are ineffective for reaching this diverse audience. Lectures and observing nights in English alone, hosted at English-speaking schools, universities, and science centers do not tend to attract a diverse audience.

For our 2012 Transit of Venus celebration, we hosted 6,000 people in a sports stadium at the University of Toronto to watch the transit. We provided outreach materials in ten languages. The original materials were written in English and the translations were sourced from among our own research group. Translations were contributed by graduate students, postdocs, faculty, and staff. We have also begun directing our outreach efforts genuinely outward, taking our events into the diverse communities of Toronto rather than hoping that members of those communities will come to us. We have found that setting up a solar telescope on a busy street corner at lunch hour is a fantastic way to reach a diverse audience and easily attracts as many people in an hour as an on-campus lecture would. When we do sidewalk astronomy of this form, we try to have materials to hand out in multiple languages.

We hope to turn this newfound capacity for outreach in multiple languages and contexts into an ongoing commitment to developing and distributing multilingual, multicultural outreach materials, starting locally and expanding globally. 